\title{
Epidemiologia, etiologia e formas clínicas das dermatofitoses em Pernambuco, 1995-2005
}

\author{
Epidemiology, etiology and clinical presentation of dermatophytosis \\ in Pernambuco, 1995-2005
}

\author{
Paula Maria Rodrigues de Barros Corrêa Damázio ${ }^{1}$, Heloisa Ramos Lacerda ${ }^{1,2}$, \\ Armando Marsden Lacerda Filho ${ }^{3,4}$, Oliane Maria Correia Magalhães ${ }^{3,4}$ e Rejane Pereira Neves ${ }^{3,4}$
}

\begin{abstract}
RESUMO
Num total de 1.238 casos de dermatofitoses, ocorridas na Cidade de Recife /PE, observou-se predomínio das tinhas de couro cabeludo (33,7\%) $e$ Trichophyton tonsurans (25,5\%), entre 1995 e 1999, enquanto as tinhas de pele glabra (35,5\%) $e$ Trichophyton rubrum (34\%) foram mais freqüentes entre 2000 e 2005. Detectou-se importante redução do Trichophyton mentagrophytes, no último período.
\end{abstract}

Palavras-chaves: Dermatofitoses. Epidemiologia. Trichophyton spp. Microsporum spp. Epidermophyton spp.

\section{ABSTRACT}

Out of a total of 1,238 cases of dermatophytosis in the city of Recife (Pernambuco), lesions of the scalp (33.7\%) and Trichophyton tonsurans (25.5\%) predominated between 1995 and 2000, while lesions of the hairless skin (35.5\%) and Trichophyton rubrum (34\%) were the most frequent between 2000 and 2005. A significant reduction in Trichophyton mentagrophytes was detected in the second of these periods.

Key-words: Dermatophytosis. Epidemiology. Trichophyton spp. Microsporum spp. Epidermophyton spp.

Vários autores têm relatado mudanças no perfil epidemiológico e nos agentes etiológicos das dermatofitoses em diferentes países. No Brasil, estas mudanças têm sido observadas nas regiões Sul, Sudeste e Centro Oeste ${ }^{3569}$. Entretanto, poucos dados do Nordeste do país foram publicados nos últimos anos ${ }^{128}$, havendo importante lacuna de informação sobre o tema na região.

Neste trabalho, foram compiladas as dermatofitoses mais comuns em pacientes atendidos no Laboratório de Micologia Médica da Universidade Federal de Pernambuco no período de janeiro de 1995 a junho de 2005 e ainda foram avaliadas as mudanças nas espécies mais prevalentes neste período, divididos de 1995 a 1999 e de 2000 a 2005.

As amostras utilizadas para o diagnóstico das dermatofitoses foram bulbo piloso, escamas epidérmicas e ungueais colhidas por escarificação utilizando bisturis previamente esterilizados. 0 exame direto foi realizado após clarificação da amostra clínica em solução aquosa a $20 \%$ de hidróxido de potássio e a espécie definida pela análise das características macro e microscópicas das culturas realizadas em meio de ágar Sabouraud adicionado de $50 \mathrm{mg} / \mathrm{L}$ de cloranfenicol.

Foram estudados 1.238 casos de dermatofitoses em 1.105 pacientes. Aspectos relacionados ao sexo e idade estão descritos na Tabela 1.

O sítio de infecção variou significativamente conforme a faixa etária. Houve um nítido predomínio da dermatofitose de couro cabeludo entre os mais jovens, ao passo que a tinha de pele glabra foi a forma mais comum entre os maiores de 20 anos (Tabela 1).

Quanto à etiologia, Trichophyton rubrum foi isolado em 331 (26,7\%) amostras, seguido por Trichophyton tonsurans em 325 (26,2\%), Microsporum canis em 229 (18,5\%), Trichophyton mentagrophytes em 223 (18\%), Epidermophyton floccosum em 80 (6,4\%), Microsporum gypseum em 33 (2,7\%) e Trichophyton violaceum em 18 (1,4\%). Microsporum audouinii foi isolado em um $(0,1 \%)$ paciente.

\footnotetext{
1. Curso de Pós-Graduação em Medicina Tropical, Centro de Ciências da Saúde, Universidade Federal de Pernambuco, Recife, PE. 2. Departamento de Medicina Clínica, Universidade Federal de Pernambuco, Recife, PE 3. Departamento de Micologia, Centro de Ciências Biológicas, Universidade Federal de Pernambuco, Recife, PE. 4. Laboratório de Micologia Médica, Centro de Ciências Biológicas, Universidade Federal de Pernambuco, Recife, PE.

Endereço para correspondência: Prof ${ }^{a}$ Heloisa Ramos Lacerda. Curso de Pós-Graduação em Medicina Tropical/UFPE. R. Prof ${ }^{a}$ Anunciada da Rocha Melo 97/501, Madalena, 50710-390 Recife, PE.

Telefax: 5581 2126-8527; 5581 9213-3885

e-mail: helramos@terra.com.br

Recebido em: 28/12/2006

Aceito em: 27/04/2007
} 


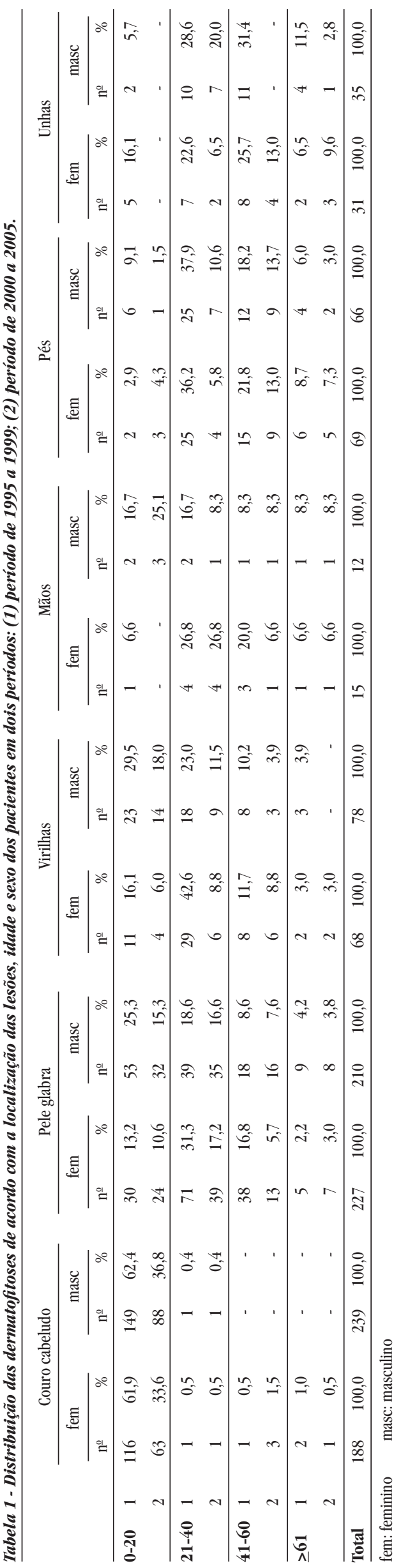

0 principal agente isolado nas amostras de pele glabra, mãos e virilhas foi o Trichophyton rubrum que totalizou 331 (26,7\%) das amostras coletadas no estudo; no couro cabeludo Trichophyton tonsurans e Microsporum canis somaram 325 (26,2\%) e $229(18,5 \%)$ do total de amostras respectivamente; nas escamas ungueais e pés predominou Trichophyton mentagrophytes que foi isolado em 223 (18\%). Dos 176 casos de tinha de couro cabeludo, causados por Microsporum canis, 68\% ocorreram nas amostras de pacientes pertencentes ao sexo masculino. Um total de 58,2\% de amostras de Epidermophyton floccosum foi isolado das dobras anatômicas.

0 período estudado foi dividido em duas partes: a primeira, entre janeiro de 1995 e dezembro de 1999, quando foram analisadas 793 amostras, tendo predominado as tinhas de couro cabeludo e o principal agente foi Trichophyton tonsurans. $\mathrm{Na}$ segunda parte, de janeiro de 2000 a junho de 2005, foram analisadas 445 amostras, tendo prevalecido as tinhas de pele glabra e Trichophyton rubrum. As espécies isoladas nos dois períodos estão demonstradas na Figura 1, estabelecendo-se uma mudança de etiologia quando se comparou os dois períodos.

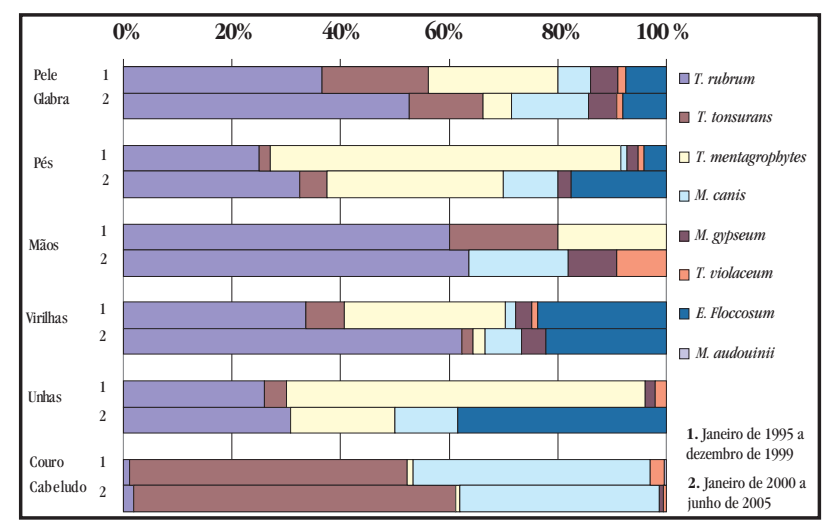

Figura 1- Relação entre espécie de dermatófito e localização da lesão, de acordo com o periodo estudado.

\section{DISCUSSÃO}

Enquanto a tinha de pele glabra foi a forma clínica mais comum, especialmente entre os maiores de 20 anos, a mão foi a localização menos acometida por dermatofitose, o que segue a tendência de outros locais do Brasil ${ }^{38}$.

Trichophyton rubrum foi a espécie mais freqüentemente isolada em nossa amostra, semelhante ao já observado em várias regiões do Brasil $^{348}$, Estados Unidos ${ }^{6}$ e em alguns países europeus ${ }^{57}$. 0 aumento do número de casos de tinha de pele glabra e mãos, localizações em que Trichophyton rubrum é o agente mais prevalente, associado a maior participação proporcional desta espécie nestas formas clínicas, resultou no aumento da prevalência deste fungo no segundo período analisado, tornando larga a diferença entre este e Trichophyton tonsurans nos últimos cinco anos. 0 aumento da prevalência de Trichophyton rubrum é observado em praticamente todo o mundo ${ }^{3569}$, entretanto os fatores responsáveis por esta elevação não são completamente compreendidos.

Ao compararmos os dois períodos do estudo, observamos um aumento do acometimento da pele glabra, couro cabeludo 
e mãos no segundo período. Constatamos também um aumento proporcional de casos devidos a Trichophyton rubrum, Microsporum canis e Trichophyton tonsurans ao passo em que houve uma diminuição significativa na detecção do Trichophyton mentagrophytes e Trichophyton violaceum.

Seguindo a tendência mundial ${ }^{9}$, o dermatófito zoofilico mais comum foi Microsporum canis. Em nosso estudo Microsporum canis passou a ser responsável por aumento no número de casos de tinha nas diversas localizações ao longo dos anos, exceto em couro cabeludo onde houve um discreto decréscimo na sua participação. Ao contrário, em São Paulo ${ }^{3}$, Goiás ${ }^{4}$, e vários países europeus ${ }^{57}$, este patógeno é o mais prevalente nas tinhas de couro cabeludo.

Trichophyton mentagrophytes foi isolado com maior frequiência em pele glabra e pé, local em que se constitui em um dos mais importantes agentes 9 . Porém, houve um declínio significativo na sua detecção em todas as localizações ao longo do período analisado.

\section{REFERÊNCIAS}

1. Brilhante RSN, Cavalcante CSP, Soares-Junior FA, Cordeiro RA, Sidrim JJC, Rocha MFG. High rate of Microsporum canis feline and canine dermatophytoses in Northeast Brazil: Epidemiological and diagnostic features. Mycopathologia 156:303-308, 2003.
2. Brilhante RSN, Cordeiro RA, Rocha MFG, Monteiro AJ, Meireles T, Sidrim JJC. Tinea capitis in a dermatology center in the city of Fortaleza, Brazil: the role of Trichophyton tonsurans. International Journal of Dermatology 43:575-579, 2004.

3. Chinelli PAV, Sofiatti AA, Nunes RS, Martins JEC. Dermatophyte agents in the city of São Paulo, from 1992 to 2002. Revista do Instituto de Medicina Tropical de São Paulo 45:259-263, 2003.

4. Costa M, Passos XS, Souza LKH, Miranda ATB, Lemos JA, Oliveira Jr JG, Silva MRR. Epidemiologia e etiologia das dermatofitoses em Goiânia, G0, Brasil. Revista da Sociedade Brasileira de Medicina Tropical 35:19-22, 2002.

5. Florencio VD, Romero-Balmas JA. Cambios em el epidemiología de las tiñas. Aspectos particulares de Andalucía. Revista Iberoamericana de Micologia 16:S3-S6, 1999.

6. Foster KW, Ghannoum MA, Elewski BE. Epidemiologic surveillance of cutaneous fungal infection in the United States from 1999 to 2002. Journal of the American Academy of Dermatology 50:748-752, 2004.

7. Frangoulis E, Athanasopoulou B, Katsambas A. Etiology of tinea capitis in Athenas, Greece - a 6-year (1996-2001) retrospective study. Mycoses 47:208-212, 2004.

8. Lima E0, Pontes ZBVS, Oliveira NMC, Carvalho MFFP, Guerra MFL, Santos JP. Frequiência das dermatofitoses em João Pessoa - Paraíba - Brasil. Anais Brasileiros de Dermatologia 74:127-132, 1999.

9. Roseeuw D. Achilles foot screening project: preliminary results of patients screened by dermatologists. Journal of the European Academy and Venereology 12(suppl 1): S6-S9, 1999 